\title{
Ethically Aligned Mobilization of Community Effort to Reposition Shared Bikes
}

\author{
Zelei Liu, ${ }^{1,3}$ Han Yu, ${ }^{2}$ Leye Wang, ${ }^{3}$ Liang Hu, ${ }^{1}$ Qiang Yang ${ }^{3}$ \\ ${ }^{1}$ College of Computer Science and Technology, Jilin University, Changchun, Jilin, China \\ ${ }^{2}$ School of Computer Science and Engineering, Nanyang Technological University, Singapore \\ ${ }^{3}$ Department of Computer Science and Engineering, Hong Kong University of Science and Technology, Hong Kong \\ liuz113@mails.jlu.edu.cn, han.yu@ntu.edu.sg, wly@cse.ust.hk, hul@jlu.edu.cn, qyang@cse.ust.hk
}

\begin{abstract}
We consider the problem of mobilizing community effort to reposition indiscriminantly parked shared bikes in urban environments through crowdsourcing. We propose an ethically aligned incentive optimization approach WSLS which maximizes the rate of success for bike repositioning while minimizing cost and prioritizing users' wellbeing. Realistic simulations based on a dataset from Singapore demonstrate that WSLS significantly outperforms existing approaches.
\end{abstract}

\section{Introduction}

Today's biking sharing systems (e.g. ofo) allow users to pick up and drop off bikes at any location, not limited to parking zones (Figure 1(a)). This flexibility has led to many cases of indiscriminant parking (Figure 1(b)) despite appeals to users to park shared bikes at designated parking zones (Figure 1(c)). This has resulted inconvenience to other road users. Existing approaches incentivizing users to change their travel plans to park bikes into parking zones (Singla et al. 2015) are not suitable for this problem.

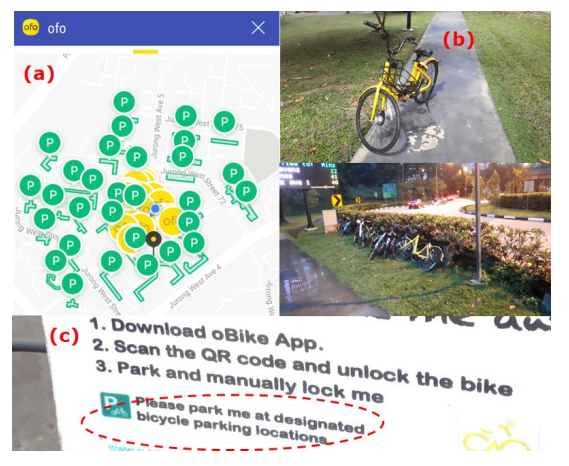

Figure 1: Examples of bike parking problems in Singapore.

To address this problem, we develop an incentive optimization approach to mobilize crowdsourcing users to reposition indiscriminately parked bikes into parking zones. We propose a revised multi-dimensional and multiple choice Knapsack formalization of this problem, and an efficient

Copyright (c) 2019, Association for the Advancement of Artificial Intelligence (www.aaai.org). All rights reserved. solution to maximize the expected number of bikes repositioned while minimizing cost and number of queries.

\section{Revised MMKP Problem Formulation}

Assuming that within a bounded area, there are $N_{S}$ shared bike parking zones. Let there be a total of $N_{B}$ indiscriminately parked bikes $\left\{b_{1}, b_{2}, \ldots, b_{N_{B}}\right\}$. The objective is to maximize the number of bikes repositioned into parking zones while staying within the resource constraints.

At any given time, there can be $N_{U}$ users, each having his own base price for repositioning a bike. A user's base price may vary at different time of a day and is related to the distance of travel needed to reposition a bike. Therefore, we divide each day into 12 time slots of 2 hours each $\left\{h_{1}, h_{2}, \ldots, h_{12}\right\}$, and consider moving distance $d$ in multiples of a fixed unit distance $d_{\text {unit }}$ (e.g. $\left.50 \mathrm{~m}\right)$. There are up to $L$ levels of movement distance $\left\{d_{1}, d_{2}, \ldots, d_{L}\right\}$, and $d_{l}=l \cdot d_{\text {unit }}$. Distances longer than $d_{L}$ are categorized as $d_{L}$. Denote user $i$ 's base price for a given distance $d_{l}$ and time slot $h_{t}$ as $c_{i}\left(d_{l}, h_{t}\right)$. We assume that the range of possible values for base prices is $c_{i}\left(d_{l}, h_{t}\right) \in\left[c_{\min }, c_{\max }\right]$, where $c_{\max } \geqslant c_{\min } \geqslant 0$. Each bikes is treated as a task, $\tau_{j}$, which needs to be delegated to a user. Each $\tau_{j}$ contains information regarding bike id $b$, the destination parking zone $z$, and distance separating the two $d_{l}, \tau_{j}=\left\{b, z, d_{l}\right\}$. We aim to reposition each bike to its nearest available parking zone.

One obvious resource constraint is the total budget, $B$. In addition, we introduce another resource constraint - total number of queries, $P T$, for all tasks. One query equals to one offer presented to one user. The main reason to introduce $P T$ is to prevent the algorithm from spamming users with lower price queries which negatively impacts user experience. By incorporating this constraint, we follow the ethically aligned design guidelines to prioritize users' wellbeing while attempting to achieve system objectives (Yu et al. 2018). We formulate our problem following the Multidimensional Multiple Choice Knapsack (MMKP) model (Hifi, Michrafy, and Sbihi 2004). We treat the decisions about each task $\tau_{j}$ as an item-set $I_{j}$ which contains $\left|I_{j}\right|$ items formed by available choices of the posted-price offer $p \in\left[c_{\min }, c_{\max }\right]$, the time slot the task is scheduled for processing $h$, and expected number of queries to be sent to nearby users $p t=\left\lceil\frac{1}{F\left(d_{l}, h, p\right)}\right\rceil$, where $F\left(d_{l}, h, p\right)$ is the cumulative probability distribution function of how likely an 
offer $p$ will be accepted given $d_{l}$ and $h$, which is to be learnt.

The original objective of MMKP is to pick exactly one item from each item-set to maximize the overall value of picked items. Since there may not always be enough resources left to pick one item from each item-set, we relax this constraint in our formulation to picking at most one item from each item-set.

\section{The WSLS Approach}

As described in our revised MMKP model, the joint decision about each task $\tau_{j}$ is an item in the item-set $I_{j}$ with $\left|I_{j}\right|$ items. The $\left|I_{j}\right|$ items can be viewed as the arms in an $\left|I_{j}\right|$-armed bandit. Based on the framework in (Hifi, Michrafy, and Sbihi 2004), we propose a Weight Sensitive Local Search (WSLS) approach. It firstly ranks all tasks in ascending order of their $d_{l}$ value. We set the time window $t w=2$, meaning that a task can be processed in either the current time slot $h_{t}$, or the next $h_{t+1}$. Then, for each task, WSLS pulls the arms in the bandit to determine the joint decision tuple $\langle p, h, p t\rangle$.

Since all successfully repositioned bikes yield the same utility, WSLS focuses on finding items consuming less resources. It contains three steps: 1) finding an initial solution selecting items which result in the minimal sum of resource consumption ratios under both $B$ and $P T$ (i.e. $\operatorname{argmin}\left\{\left(\frac{p}{B}+\frac{p t}{P T}\right) \mid h\right\}$ for every task $\left.\left.\tau_{j}\right) ; 2\right)$ adjusting $\langle p, h, p t\rangle \in I_{j}$

the choices of items based on resource violations to find a feasible solution (some selected item tuples may need to set to $\langle 0,0,0\rangle$ ); and 3) swapping items through local search by eliminating items 'dominated' by others (Kellerer, Pferschy, and Pisinger 2004) to improve solution optimality.

\section{Experimental Evaluation}

In order to evaluate the proposed WSLS approach, we built a simulator based on a real-world dataset from Singapore containing location information of designated parking zones (https://data.gov.sg/dataset/lta-parking-standards-zone).

The simulator also generates the locations of the bikes and the start locations for the user agents to create an evaluation environment.

We select four approaches as baselines for comparison with WSLS in the experiments: 1) Random, which randomly adjusts offer prices to query nearby users until someone accepts the task; 2) Greedy, which follows the greedy approach in (Auer, Cesa-Bianchi, and Fischer 2002) to adjust the offer prices; 3) Upper Confidence Bound (UCB), which follows the UCB approach in (Auer, Cesa-Bianchi, and Fischer 2002) to adjust the offer prices; and 4) Knapsack-based upper confidence bound exploration and exploitation (KUBE), which follows the approach in (Tran-Thanh et al. 2012) to adjust the offer prices. We set $N_{B}=10,000$ and vary $N_{U}$ from 5,000 to 15,000 . Under each configuration, the simulation was run for 10,000 epoches. Prices are represented in the form of points in the simulation, and a user agent only accepts an offer if it is equal to or higher than its base price.

Figure 2 shows the results. The average percentage of bikes repositioned is averaged over all configurations and epoches, whereas the other two metrics are averaged over all configurations, epoches and bikes. It can be observed that WSLS achieves the highest percentage of bikes repositioned, beating the best performing baseline approach, KUBE, by $34 \%$. It achieves the second lowest average offer price among all approaches, $17 \%$ higher than that of Greedy. However, WSLS only needs to query half as many users as Greedy does. Overall, WSLS achieves the most desirable trade-off among the three evaluation metrics.

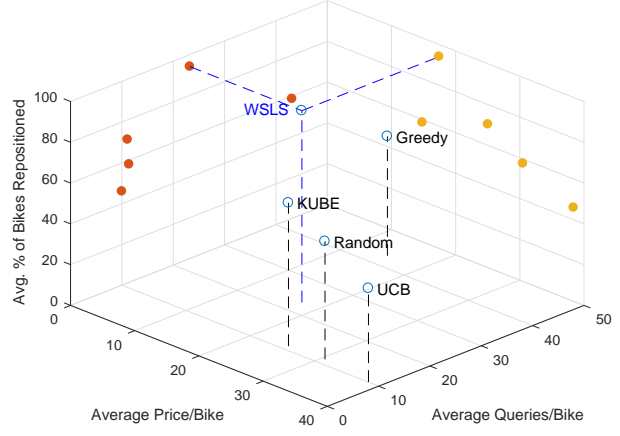

Figure 2: Experimental Results.

\section{Conclusions and Future Work}

In this paper, we proposed WSLS which jointly optimizes the offer price, the scheduled execution time, and the task query rounds in order to efficiently mobile community effort to reposition indiscriminantly parked shared bikes through crowdsourcing. We are developing a mobile app based on this approach, and working with community organizations in order to study how such a platform can be used to engage senior citizens to participate for the purpose of productive aging while achieving our original objective.

\section{Acknowledgements}

This research is supported by Nanyang Technological University, Nanyang Assistant Professorship (NAP).

\section{References}

Auer, P.; Cesa-Bianchi, N.; and Fischer, P. 2002. Finite-time analysis of the multiarmed bandit problem. Machine Learning 47(23):235-256.

Hifi, M.; Michrafy, M.; and Sbihi, A. 2004. Heuristic algorithms for the multiple-choice multidimensional knapsack problem. Journal of the Operational Research Society 55(12):1323-1332.

Kellerer, H.; Pferschy, U.; and Pisinger, D. 2004. Knapsack Problems.

Singla, A.; Santoni, M.; Bartók, G.; Mukerji, P.; Meenen, M.; and Krause, A. 2015. Incentivizing users for balancing bike sharing systems. In $A A A I, 723-729$.

Tran-Thanh, L.; Chapman, A. C.; Rogers, A.; and Jennings, N. R. 2012. Knapsack based optimal policies for budget-limited multiarmed bandits. In AAAI, 1134-1140.

Yu, H.; Shen, Z.; Miao, C.; Leung, C.; Lesser, V. R.; and Yang, Q. 2018. Building ethics into artificial intelligence. In IJCAI, 55275533 